\title{
Mortalidad por cáncer en Trujillo: 2003-2010
}

\section{Cancer mortality in Trujillo: 2003-2010}

\section{Pedro Albújar-Baca ${ }^{1}$}

1. Médico Cirujano, AnatomoPatólogo. AE Academia Nacional de Medicina. Ex Director del Registro de Cáncer de Trujillo.

\section{ABSTRACT}

Oвjective. To describe the epidemiological characteristics of cancer mortality in Metropolitan Trujillo in the 20032010 period. Material AND MetHOds. This is an observational retrospective and descriptive study using as database all death certificates with cancer as basic cause of death and the 2007 official population census data. The topographic distribution of cancer diagnoses were classified according to the 10th Revision of the International Classification of Diseases. The frequency and age standardized mortality rates were calculated. REsults. Cancer was the cause of death of 4200 persons, $44 \%$ men and $56 \%$ women. The average annual cancer death was 525. The most frequent cancer sites were stomach, cervix uteri and prostate. Three out of five deaths occurred in patients older than 65 years. Among males the leading causes of cancer deaths were tumors of stomach, prostate and lung whereas in females were cervix uteri, stomach and breast. Childhood mortality was 1,5\% of all cancer deaths, leukemia, central nervous system tumors and lymphomas were the principal cause of cancer death at this age group.

KeY WORDs: Neoplams; Mortality: Peru. grupo de edad

Palabras clave: Neoplasias; Mortalidad; Perú. 


\section{INTRODUCCIÓN}

Los estudios epidemiológicos de mortalidad constituyen un importante instrumento de evaluación de la efectividad de los programas de prevención y control del cáncer. El análisis permite identificar cambios significativos y focalizar las conclusiones en las causas más prevenibles.

Según la Agencia Internacional de Investigación en Cáncer (IARC), ${ }^{1}$ en el año 2008, 7,6 millones personas murieron de cáncer en el mundo, el $63 \%$ en las regiones económicamente menos desarrolladas. Un informe de la OPS/OMS ${ }^{2}$ revela que el $50 \%$ de las muertes en las Américas ocurren en América Latina y el Caribe que concentra el 63\% de la población del continente. El año 2007 la tasa de mortalidad por cáncer en Perú fue 107,9 por 100000 habitantes, segunda causa de muerte después de las enfermedades transmisibles. ${ }^{3}$ En Trujillo, ${ }^{4}$ el cáncer fue la primera causa de muerte entre 3886 defunciones.

El presente estudio tiene como objetivos medir la magnitud, características y distribución de las defunciones por cáncer registradas en el área metropolitana de la ciudad de Trujillo en un período de ocho años.

\section{MATERIAL Y MÉTODO}

El estudio es de tipo observacional descriptivo retrospectivo. Los datos se obtuvieron de todos los certificados de defunción con diagnóstico de cáncer como causa básica de muerte proporcionados por la Dirección Regional de Salud de La Libertad. La recopilación cubrió un período de ocho años, 2003 a 2010. Se registraron las siguientes variables: edad, sexo, localización y tipo de cáncer. El área geográfica del estudio fue Trujillo Metropolitano que comprende los distritos de Trujillo, La Esperanza, Florencia de Mora, El Porvenir y Víctor Larco, con una población de 730913 habitantes. Para la clasificación y estandarización de los distintos tipos de cáncer se utilizó la Décima Revisión de la Clasificación Internacional de Enfermedades, Capítulo II, Neoplasias, que comprende los códigos del $\mathrm{COO}$ al C96. Se calculó la frecuencia porcentual, las tasas específicas por grupo de edad según el método directo y las tasas estandarizadas de mortalidad
(TEM) a la población mundial por 100 000. En 29 certificados de defunción no constaba la edad del fallecido y en 116 tampoco la localización primaria del tumor.

\section{RESULTADOS}

La información pormenorizada de la mortalidad por cáncer se presenta en la Tabla 1 para hombres y en la Tabla 2 para mujeres. En los hombres el ascenso de la mortalidad es a partir de los 45 años, en las mujeres a partir de los 40 años.

En el período fallecieron por cáncer 4200 personas, con una media anual de 525 defunciones. Fallecieron de cáncer más mujeres 2341 (55,7\%) que hombres 1859 (44,3\%), con tasas estandarizadas de mortalidad de 79,0 y 73,4 respectivamente. Ver Tabla 3.

Las cinco localizaciones con mayor mortalidad por cáncer fueron estómago, cuello uterino, próstata, colon- recto y pulmón, en conjunto representaron $48 \%$ de todas las muertes por cáncer como se aprecia en la Tabla 4. Anualmente murieron 90 personas por cáncer de estómago, 50 de cuello uterino, 38 de próstata, 37 colorrectal, 36 pulmón y 30 de mama.

Por grupos de edad según Tabla 5. La mayor mortalidad por cáncer, $60 \%$, se produjo en mayores de 65 años, 39\% entre los 15 y 64 años y $1.5 \%$ en menores de 15 años.

Los cánceres que ocasionaron mayor número de muertes en hombres se localizaron en estómago, próstata y pulmón que en conjunto representaron el $44 \%$. En las mujeres el cáncer de cuello uterino, estómago y mama, juntos representaron el $42,5 \%$ de la mortalidad por cáncer. Ver Tabla 6.

El $40 \%$ de la mortalidad general por cáncer afectó el aparato digestivo, $12,4 \%$ el aparato genital femenino, $10,2 \%$ el tejido linfohematopoyético, $7,7 \%$ el aparato genital masculino y $7,6 \%$ el aparato respiratorio.

La principal localización de la mortalidad por cáncer fue el estómago. El 58\% de muertes se produjo después de los 65 años. La relación de mortalidad hombre mujer fue 1:1. En los hombres ocupó el primer lugar de mortalidad por cáncer con TEM 14,6, después de los 65 años pasó al 


\section{Anta Méäica Pernana}

Tabla 1. Número de muertes por cáncer según localización y grupo de edad, en hombres

\begin{tabular}{|c|c|c|c|c|c|c|c|c|c|c|c|c|c|c|c|c|c|c|c|c|c|}
\hline IE-10 & ocalización & dad & 0 & & & & & $25-$ & $30-$ & & & & & $55-$ & $60-$ & $65-$ & & & & & \\
\hline All & Todas las localizaciones & 11 & 7 & 13 & 14 & 11 & 21 & 18 & 31 & 46 & 53 & 68 & 99 & 105 & 188 & 190 & 258 & 255 & 223 & 248 & 1859 \\
\hline $\mathrm{CO1}-\mathrm{CO} 2$ & Lengua & 0 & 0 & 0 & 0 & 0 & 0 & 0 & 0 & 0 & 0 & 0 & 1 & 0 & 0 & 1 & 0 & 1 & 0 & 0 & 3 \\
\hline $\mathrm{CO3-C06}$ & Cavidad oral & 0 & 0 & 0 & 0 & 0 & 1 & 0 & 1 & 0 & 1 & 1 & 1 & 0 & 1 & 1 & 1 & 1 & 1 & 1 & 11 \\
\hline $\mathrm{CO}-\mathrm{COB}$ & Glándulas salivales & 0 & 0 & 0 & 0 & 0 & 0 & 0 & 0 & 0 & 0 & 0 & 0 & 0 & 0 & 0 & 0 & 0 & 1 & 0 & 1 \\
\hline C09-C11 & Faringe & 0 & 0 & 0 & 0 & 0 & 0 & 0 & 0 & 0 & 1 & 0 & 0 & 1 & 1 & 0 & 1 & 0 & 0 & 0 & 4 \\
\hline $\mathrm{C} 15$ & Esófago & 0 & 0 & 0 & 0 & 0 & 0 & 0 & 0 & 1 & 0 & 1 & 0 & 1 & 3 & 1 & 4 & 3 & 5 & 8 & 27 \\
\hline $\mathrm{C} 16$ & Estómago & 2 & 0 & 0 & 0 & 0 & 1 & 3 & 8 & 14 & 6 & 17 & 30 & 19 & 49 & 35 & 52 & 40 & 45 & 39 & 360 \\
\hline $\mathrm{C} 17$ & Intestino delgado & 0 & 0 & 0 & 0 & 0 & 0 & 0 & 0 & 0 & 1 & 2 & 4 & 1 & 4 & 1 & 5 & 2 & 4 & 2 & 26 \\
\hline $\mathrm{C} 18-\mathrm{C} 20$ & Colon, recto & 1 & 0 & 0 & 0 & 0 & 0 & 1 & 1 & 0 & 9 & 4 & 5 & 10 & 12 & 10 & 18 & 24 & 16 & 8 & 119 \\
\hline $\mathrm{C} 22$ & Hígado & 0 & 0 & 0 & 0 & 0 & 1 & 0 & 1 & 5 & 2 & 2 & 6 & 13 & 9 & 14 & 21 & 16 & 8 & 10 & 108 \\
\hline $\mathrm{C} 23-\mathrm{C} 24$ & Vesícula biliar - vías biliares & 0 & 0 & 0 & 0 & 0 & 0 & 0 & 1 & 3 & 2 & 1 & 0 & 1 & 6 & 5 & 10 & 10 & 8 & 4 & 51 \\
\hline $\mathrm{C} 25$ & Páncreas & 1 & 0 & 0 & 0 & 0 & 1 & 0 & 2 & 2 & 1 & 6 & 7 & 3 & 10 & 10 & 17 & 19 & 9 & 9 & 97 \\
\hline C30-C31 & Cavidad nasal & 0 & 0 & 0 & 0 & 0 & 0 & 0 & 0 & 0 & 0 & 1 & 0 & 0 & 0 & 0 & 1 & 1 & 1 & 0 & 4 \\
\hline C32 & Laringe & 0 & 0 & 0 & 0 & 0 & 0 & 0 & 0 & 0 & 0 & 0 & 1 & 0 & 2 & 1 & 1 & 0 & 1 & 4 & 10 \\
\hline C34 & Pulmón & 0 & 0 & 0 & 0 & 0 & 1 & 0 & 1 & 2 & 3 & 4 & 10 & 9 & 16 & 16 & 26 & 24 & 17 & 16 & 145 \\
\hline C40-C41 & Hueso & 0 & 0 & 1 & 1 & 0 & 2 & 2 & 4 & 2 & 1 & 2 & 3 & 2 & 0 & 4 & 1 & 0 & 3 & 1 & 29 \\
\hline C43 & Piel, melanoma & 0 & 0 & 0 & 0 & 0 & 0 & 0 & 0 & 0 & 0 & 0 & 0 & 0 & 2 & 3 & 2 & 3 & 1 & 5 & 16 \\
\hline C44 & Piel, sin melanoma & 0 & 0 & 0 & 0 & 0 & 0 & 0 & 0 & 0 & 0 & 0 & 1 & 0 & 1 & 0 & 0 & 0 & 0 & 2 & 4 \\
\hline C49 & Tejidos conectivo y blandos & 0 & 1 & 0 & 1 & 1 & 0 & 0 & 2 & 2 & 4 & 1 & 2 & 1 & 4 & 2 & 4 & 4 & 3 & 3 & 35 \\
\hline $\mathrm{C} 60$ & Pene & 0 & 0 & 0 & 0 & 0 & 0 & 0 & 0 & 1 & 0 & 0 & 0 & 0 & 0 & 1 & 1 & 0 & 1 & 1 & 5 \\
\hline C61 & Próstat & 3 & 0 & 0 & 0 & 0 & 0 & 0 & 0 & 0 & 0 & 3 & 4 & 8 & 17 & 28 & 37 & 60 & 66 & 80 & 306 \\
\hline C62 & Testículo & 0 & 1 & 0 & 0 & 1 & 3 & 3 & 1 & 0 & 0 & 0 & 1 & 1 & 0 & 0 & 0 & 1 & 0 & 0 & 12 \\
\hline C64 & Riñón & 0 & 1 & 0 & 0 & 0 & 1 & 0 & 0 & 0 & 0 & 0 & 2 & 5 & 8 & 12 & 11 & 4 & 4 & 1 & 49 \\
\hline C67 & Vejiga urinaria & 0 & 0 & 0 & 0 & 1 & 0 & 0 & 0 & 0 & 0 & 0 & 1 & 0 & 1 & 2 & 6 & 7 & 5 & 10 & 33 \\
\hline C69 & Ojo & 0 & 0 & 0 & 0 & 0 & 0 & 0 & 0 & 0 & 0 & 0 & 0 & 0 & 1 & 0 & 0 & 0 & 0 & 0 & 1 \\
\hline $\mathrm{C} 70-\mathrm{C} 72$ & Cerebro, médula espinal & 0 & 2 & 4 & 2 & 1 & 1 & 4 & 3 & 4 & 8 & 4 & 7 & 4 & 10 & 4 & 8 & 5 & 4 & 3 & 78 \\
\hline $\mathrm{C} 73$ & Tiroides & 0 & 0 & 0 & 0 & 0 & 0 & 0 & 0 & 0 & 1 & 2 & 0 & 0 & 0 & 2 & 0 & 0 & 0 & 6 & 11 \\
\hline C81-C85 & Ganglio linfático & 1 & 0 & 0 & 3 & 1 & 2 & 5 & 2 & 4 & 6 & 3 & 5 & 10 & 15 & 16 & 6 & 12 & 9 & 11 & 111 \\
\hline C90 & Mieloma múltiple & 1 & 0 & 0 & 0 & 0 & 0 & 0 & 0 & 0 & 0 & 1 & 2 & 3 & 4 & 5 & 7 & 5 & 1 & 2 & 31 \\
\hline C91-C95 & Leucemia & 0 & 2 & 8 & 6 & 6 & 7 & 0 & 3 & 5 & 4 & 9 & 5 & 4 & 5 & 9 & 7 & 5 & 3 & 9 & 97 \\
\hline $\mathrm{C} 80$ & Sitio primario desconocido & 2 & 0 & 0 & 1 & 0 & 0 & 0 & 1 & 1 & 3 & 4 & 1 & 9 & 7 & 7 & 11 & 8 & 7 & 13 & 75 \\
\hline
\end{tabular}

segundo lugar luego del cáncer de próstata. En el sexo femenino el cáncer gástrico ocupó el segundo lugar de mortalidad con TEM 11,3 después de los 65 años fue la primera causa de muerte.

El cáncer de cuello uterino se situó en el segundo lugar dentro de mortalidad general por cáncer. Fue la primera causa de muerte por cáncer en la mujer con TEM 14,0. El 59\% de las muertes se ocurrieron entre los 30 y 60 años.
El cáncer de próstata ocupó el tercer lugar en la mortalidad global por cáncer. Entre los hombres fue la segunda causa de muerte por cáncer con TEM 14,6. El $88 \%$ de los decesos se produjeron después de los 65 años.

El cáncer de colorrectal se ubicó en cuarto lugar de la mortalidad general por cáncer, el $70 \%$ de las muertes afectaron a pacientes mayores de 65 años. La TEM fue mayor en mujeres, 5,7, que en hombres, 4,7. 


\begin{tabular}{|c|c|c|c|c|c|c|c|c|c|c|c|c|c|c|c|c|c|c|c|c|c|}
\hline \multicolumn{2}{|c|}{ CIE-10ma localización } & edad & & \multicolumn{2}{|c|}{ 5- 10} & \multicolumn{2}{|c|}{ 15- 20} & \multirow{2}{*}{$\begin{array}{l}25- \\
23\end{array}$} & \multirow{2}{*}{$\begin{array}{l}30- \\
43\end{array}$} & \multirow{2}{*}{$\begin{array}{l}35- \\
53\end{array}$} & \multirow{2}{*}{$\begin{array}{l}40- \\
109\end{array}$} & \multirow{2}{*}{$\begin{array}{l}45- \\
151\end{array}$} & \multirow{2}{*}{$\begin{array}{l}50- \\
164\end{array}$} & \multirow{2}{*}{$\begin{array}{l}55- \\
192\end{array}$} & \multirow{2}{*}{$\begin{array}{l}60- \\
212\end{array}$} & \multirow{2}{*}{$\begin{array}{l}65- \\
234\end{array}$} & \multirow{2}{*}{$\begin{array}{l}70- \\
271\end{array}$} & \multirow{2}{*}{$\begin{array}{l}75- \\
269\end{array}$} & \multirow{2}{*}{$\begin{array}{l}80- \\
251\end{array}$} & \multirow{2}{*}{$\begin{array}{l}85+ \\
288\end{array}$} & \multirow{2}{*}{$\begin{array}{l}\text { Total } \\
2341\end{array}$} \\
\hline All & Todas las localizaciones & 16 & 8 & 7 & 14 & 22 & 14 & & & & & & & & & & & & & & \\
\hline $\mathrm{CO1}-\mathrm{CO}$ & Lengua & 0 & 0 & 0 & 0 & 0 & 0 & 0 & 0 & 0 & 0 & 0 & 0 & 0 & 1 & 0 & 2 & 1 & 0 & 0 & 4 \\
\hline $\mathrm{CO3}-\mathrm{CO}$ & Cavidad oral & 0 & 0 & 0 & 0 & 0 & 1 & 0 & 1 & 0 & 0 & 1 & 2 & 0 & 1 & 3 & 2 & 0 & 5 & 7 & 23 \\
\hline C07-C09 & Glándulas salivales & 0 & 0 & 0 & 0 & 0 & 0 & 0 & 0 & 0 & 0 & 0 & 0 & 0 & 0 & 0 & 0 & 0 & 0 & 1 & 1 \\
\hline $\mathrm{CO9}-\mathrm{C} 12$ & Faringe & 0 & 0 & 0 & 0 & 0 & 0 & 0 & 0 & 0 & 0 & 0 & 0 & 0 & 1 & 0 & 0 & 0 & 1 & 0 & 2 \\
\hline $\mathrm{C} 15$ & Esófago & 0 & 0 & 0 & 0 & 0 & 0 & 0 & 0 & 0 & 1 & 0 & 0 & 0 & 2 & 1 & 2 & 2 & 1 & 2 & 11 \\
\hline $\mathrm{C} 16$ & Estómago & 4 & 0 & 0 & 0 & 0 & 0 & 4 & 10 & 8 & 18 & 20 & 27 & 25 & 34 & 27 & 35 & 45 & 39 & 58 & 354 \\
\hline $\mathrm{C} 17$ & Intestino delgado & 0 & 0 & 0 & 0 & 0 & 0 & 0 & 0 & 0 & 0 & 0 & 0 & 1 & 1 & 3 & 5 & 0 & 0 & 5 & 15 \\
\hline $\mathrm{C} 18-\mathrm{C} 21$ & Colon, recto & 0 & 0 & 0 & 0 & 0 & 0 & 1 & 0 & 1 & 3 & 8 & 8 & 12 & 12 & 20 & 27 & 25 & 28 & 36 & 181 \\
\hline $\mathrm{C} 22$ & Ano & 0 & 0 & 0 & 0 & 0 & 0 & 0 & 0 & 0 & 0 & 0 & 0 & 0 & 0 & 1 & 0 & 0 & 2 & 0 & 3 \\
\hline $\mathrm{C} 23$ & Hígado & 1 & 1 & 0 & 1 & 0 & 0 & 2 & 0 & 1 & 2 & 3 & 9 & 6 & 8 & 15 & 14 & 28 & 29 & 21 & 141 \\
\hline $\mathrm{C} 23-\mathrm{C} 25$ & Vesícula biliar, vías biliares & 1 & 0 & 0 & 0 & 0 & 0 & 0 & 1 & 1 & 1 & 1 & 4 & 13 & 8 & 16 & 17 & 9 & 15 & 9 & 96 \\
\hline $\mathrm{C} 26$ & Páncreas & 0 & 0 & 0 & 0 & 0 & 0 & 1 & 0 & 0 & 6 & 3 & 2 & 5 & 11 & 16 & 10 & 12 & 13 & 15 & 94 \\
\hline C30-C32 & Cavidad nasal & 0 & 0 & 0 & 0 & 1 & 0 & 0 & 0 & 0 & 0 & 0 & 0 & 1 & 0 & 1 & 0 & 2 & 1 & 0 & 6 \\
\hline C33 & Laringe & 0 & 0 & 0 & 0 & 0 & 0 & 0 & 0 & 0 & 0 & 0 & 0 & 0 & 1 & 0 & 1 & 2 & 0 & 1 & 5 \\
\hline C35 & Pulmón & 0 & 0 & 0 & 0 & 0 & 1 & 0 & 0 & 5 & 2 & 3 & 11 & 14 & 12 & 18 & 24 & 20 & 20 & 19 & 149 \\
\hline C40-C42 & Hueso & 0 & 0 & 0 & 1 & 1 & 2 & 1 & 1 & 2 & 0 & 2 & 2 & 0 & 0 & 4 & 1 & 4 & 2 & 3 & 26 \\
\hline C44 & Piel, melanoma & 1 & 0 & 0 & 0 & 0 & 0 & 0 & 1 & 1 & 0 & 0 & 0 & 1 & 0 & 3 & 0 & 1 & 1 & 6 & 15 \\
\hline C45 & Piel, sin melanoma & 0 & 0 & 0 & 0 & 0 & 0 & 0 & 0 & 0 & 0 & 0 & 0 & 0 & 0 & 0 & 0 & 0 & 0 & 2 & 2 \\
\hline C49 & Retroperitoneo & 0 & 0 & 0 & 0 & 0 & 0 & 0 & 0 & 0 & 0 & 0 & 0 & 0 & 0 & 0 & 0 & 1 & 0 & 0 & 1 \\
\hline $\mathrm{C} 50$ & Tejidos conectivo y blandos & 0 & 0 & 0 & 0 & 1 & 0 & 1 & 0 & 0 & 2 & 1 & 2 & 5 & 3 & 3 & 1 & 3 & 0 & 1 & 23 \\
\hline $\mathrm{C} 50$ & Mama & 1 & 0 & 0 & 0 & 1 & 1 & 1 & 5 & 6 & 15 & 34 & 25 & 32 & 20 & 23 & 27 & 16 & 13 & 16 & 236 \\
\hline C51-C52 & Vagina, vulva & 1 & 0 & 0 & 0 & 0 & 0 & 0 & 0 & 0 & 0 & 0 & 0 & 0 & 0 & 1 & 0 & 1 & 0 & 1 & 4 \\
\hline C53 & Cuello uterino & 2 & 0 & 0 & 0 & 1 & 1 & 6 & 13 & 18 & 32 & 52 & 39 & 41 & 41 & 28 & 43 & 33 & 20 & 31 & 401 \\
\hline C54 & Cuerpo uterino & 0 & 0 & 0 & 0 & 0 & 0 & 0 & 0 & 0 & 0 & 1 & 3 & 1 & 6 & 2 & 0 & 4 & 1 & 0 & 18 \\
\hline C56 & Ovario & 0 & 0 & 0 & 2 & 3 & 0 & 1 & 2 & 3 & 12 & 9 & 6 & 6 & 11 & 8 & 8 & 7 & 8 & 11 & 97 \\
\hline $\mathrm{C} 65$ & Riñón & 1 & 0 & 0 & 0 & 0 & 0 & 0 & 0 & 0 & 2 & 2 & 4 & 1 & 4 & 4 & 4 & 4 & 4 & 2 & 32 \\
\hline C68 & Vejiga urinaria & 0 & 0 & 0 & 0 & 0 & 0 & 0 & 0 & 0 & 0 & 0 & 0 & 1 & 2 & 0 & 3 & 2 & 2 & 7 & 17 \\
\hline C69 & Uretra & 0 & 0 & 0 & 0 & 0 & 0 & 0 & 0 & 0 & 0 & 0 & 0 & 0 & 1 & 0 & 0 & 0 & 0 & 0 & 1 \\
\hline $\mathrm{C} 70$ & Ojo & 0 & 2 & 0 & 0 & 0 & 0 & 0 & 0 & 0 & 0 & 0 & 0 & 0 & 0 & 1 & 0 & 0 & 0 & 0 & 3 \\
\hline C70-C73 & Cerebro, médula espinal & 1 & 2 & 1 & 4 & 3 & 2 & 0 & 3 & 1 & 7 & 1 & 4 & 3 & 7 & 6 & 7 & 7 & 5 & 5 & 69 \\
\hline $\mathrm{C} 74$ & Tiroides & 0 & 0 & 0 & 0 & 0 & 0 & 0 & 1 & 1 & 0 & 0 & 2 & 1 & 4 & 3 & 8 & 3 & 7 & 1 & 31 \\
\hline C81-C86 & Ganglio linfático & 1 & 0 & 2 & 0 & 2 & 1 & 0 & 1 & 0 & 2 & 2 & 4 & 5 & 7 & 11 & 8 & 13 & 15 & 10 & 84 \\
\hline C91 & Mieloma múltiple & 1 & 0 & 0 & 0 & 0 & 0 & 0 & 0 & 0 & 0 & 0 & 1 & 2 & 3 & 1 & 6 & 2 & 0 & 3 & 19 \\
\hline C91-C95 & Leucemia & 1 & 2 & 3 & 6 & 9 & 5 & 3 & 4 & 4 & 2 & 3 & 3 & 6 & 8 & 6 & 5 & 8 & 6 & 2 & 86 \\
\hline $\mathrm{C} 80$ & Sitio primario desconocido & 0 & 1 & 1 & 0 & 0 & 0 & 2 & 0 & 1 & 2 & 5 & 6 & 10 & 3 & 9 & 11 & 14 & 13 & 13 & 91 \\
\hline
\end{tabular}

El cáncer de pulmón ocupó el quinto puesto en la mortalidad general por cáncer. El $68 \%$ de las muertes ocurrieron en pacientes mayores de 65 años. En hombres ocupó el tercer lugar con TEM 5,9 y en mujeres el quinto lugar con TEM 4,9.

El cáncer de mama ocupó el séptimo lugar en la mortalidad general por cáncer y el tercero en la serie femenina con TEM 11,3. La mortalidad más alta, 53\%, se registró entre los 40 y 60 años.

La mortalidad por cáncer en la niñez según la Tabla 7 representó 1,5\% de la mortalidad total por cáncer. La relación niño/niña fue 1/1. Las leucemias fueron la principal causa de muerte por cáncer en la niñez a ellas siguieron los tumores del sistema 


\begin{tabular}{|c|c|c|c|c|c|c|}
\hline \multirow[t]{2}{*}{ CIE-10 Localización } & \multicolumn{3}{|c|}{ Hombres } & \multicolumn{3}{|c|}{ Mujeres } \\
\hline & $\mathrm{N}$ & $\%$ & TEM & $\mathrm{N}$ & $\%$ & TEM \\
\hline C01-C03 Lengua & 3 & 0,2 & 0,1 & 4 & 0,2 & 0,2 \\
\hline C03-C07 Cavidad oral & 11 & 0,6 & 0,4 & 23 & 0,9 & 0,7 \\
\hline C07-C09 Glándulas salivales & 1 & 0,1 & 0,3 & 1 & 0,4 & 0,2 \\
\hline C09-C12 Faringe & 4 & 0,2 & 0,2 & 2 & 0,1 & 0,7 \\
\hline C16 Esófago & 27 & 1,5 & 1,0 & 11 & 0,5 & 0,4 \\
\hline Estómago & 360 & 19,4 & 14,6 & 354 & 15,1 & 11,3 \\
\hline Intestino delgado & 26 & 1,4 & 1,1 & 15 & 0,6 & 0,5 \\
\hline C18-C21 Colon, recto & 119 & 6,4 & 4,7 & 181 & 7,7 & 5,7 \\
\hline C22 Ano & 0 & 0,0 & 0,0 & 3 & 0,1 & 0,1 \\
\hline C23 Hígado & 108 & 5,8 & 4,5 & 141 & 6,0 & 4,3 \\
\hline $\begin{array}{c}\text { C23-C25 Vesícula biliar, } \\
\text { vías biliares }\end{array}$ & 51 & 2,7 & 2,0 & 96 & 4,1 & 3,3 \\
\hline C26 Páncreas & 97 & 5,2 & 3,9 & 94 & 4,0 & 3,1 \\
\hline C30-C32 Cavidad nasal & 4 & 0,2 & 0,2 & 6 & 0,3 & 0,2 \\
\hline C33 Laringe & 10 & 0,5 & 0,4 & 5 & 0,2 & 0,2 \\
\hline C35 Pulmón & 145 & 7,8 & 5,9 & 149 & 6,4 & 4,9 \\
\hline C40-C42 Hueso & 29 & 1,6 & 1,1 & 26 & 1,1 & 0,8 \\
\hline C44 Piel, melanoma & 16 & 0,9 & 0,6 & 15 & 0,6 & 0,4 \\
\hline Piel, no melanoma & 4 & 0,2 & 0,2 & 2 & 0,1 & 0,4 \\
\hline Retroperitoneo & 0 & 0,0 & 0,0 & 1 & 0,4 & 0,3 \\
\hline $\begin{array}{l}\text { Tejidos conectivo } \\
\text { y blandos }\end{array}$ & 35 & 1,8 & 1,4 & 23 & 0,9 & 2,2 \\
\hline Mama & 0 & - & - & 236 & 10,1 & 8,4 \\
\hline C51-C52 Vagina, vulva & - & - & - & 4 & 0,2 & 0,1 \\
\hline Cuello uterino & - & - & - & 401 & 17,1 & 14,0 \\
\hline Cuerpo uterino & - & - & - & 18 & 0,7 & 0,7 \\
\hline Ovario & - & - & - & 97 & 4,1 & 3,3 \\
\hline Pene & 5 & 0,3 & 0,2 & - & - & - \\
\hline Próstata & 306 & 16,5 & 10,9 & - & - & - \\
\hline Testículo & 12 & 0,7 & 0,4 & - & - & - \\
\hline Riñón & 49 & 2,6 & 2,2 & 32 & 1,4 & 1,1 \\
\hline Vejiga urinaria & 33 & 1,8 & 1,2 & 17 & 0,7 & 0,5 \\
\hline Uretra & 0 & 0,0 & 0,0 & 1 & 0,4 & 0,5 \\
\hline $\mathrm{C} 70$ & 1 & 0,1 & 0,1 & 3 & 0,1 & 0,2 \\
\hline $\begin{array}{l}\text { C70-C73 Cerebro, } \\
\text { médula espinal }\end{array}$ & 78 & 4,2 & 3,2 & 69 & 2,9 & 2,3 \\
\hline C74 Tiroides & 11 & 0,6 & 0,4 & 31 & 1,3 & 1,1 \\
\hline C81-C86 Ganglio linfático & 111 & 5,9 & 4,5 & 84 & 3,6 & 2,7 \\
\hline C91 Mieloma múltiple & 31 & 1,7 & 1,3 & 19 & 0,8 & 0,7 \\
\hline C91-C95 Leucemia & 97 & 5,2 & 3,8 & 86 & 3,7 & 2,8 \\
\hline $\begin{array}{ll}\text { C80 Sitio primario } \\
\text { desconocido }\end{array}$ & 75 & 4,0 & 2,9 & 91 & 3,9 & 2,9 \\
\hline
\end{tabular}

Todas las localizaciones $1859 \quad 100 \quad 73,1 \quad 2341 \quad 10079$

Tabla 4. Frecuencia de las diez principales localizaciones de
muertes por cáncer, ambos sexos

nervioso central y los linfomas. Los tumores del sistema linfohematopoyético, leucemias y linfomas juntos, significaron el $50,8 \%$.de todas las muertes por cáncer en la niñez.

\section{DISCUSIÓN}

En Trujillo, el cáncer ha alcanzado el nivel de problema de salud pública. En 1980 ocupaba el quinto lugar entre todas las causas de muerte, en el año 2010 se ubicó en el primer lugar.

Dentro de la mortalidad global por cáncer, el cáncer de estómago fue la principal causa de muerte en Trujillo, ${ }^{5}$ Lima $^{6}$ y Arequipa. ${ }^{7}$ A nivel sudamericano, Quito ${ }^{8}$ y Cali ${ }^{9}$ registraron también el cáncer de estómago como la primera causa de mortalidad por cáncer a diferencia de la vertiente del Atlántico, Argentina ${ }^{10}$ y Uruguay, ${ }_{11}^{11}$ cuyas tasas son bajas.

\begin{tabular}{|c|c|c|c|c|c|c|}
\hline \multirow[t]{2}{*}{ Edad (años) } & \multicolumn{2}{|c|}{ Hombres } & \multicolumn{2}{|c|}{ Mujeres } & \multicolumn{2}{|c|}{ Total } \\
\hline & $n$ & $\%$ & $n$ & $\%$ & $n$ & $\%$ \\
\hline $0-14$ & 34 & 1,8 & 29 & 1,2 & 63 & 1,5 \\
\hline $15-44$ & 180 & 9,7 & 264 & 11,4 & 444 & 10,6 \\
\hline $45-64$ & 460 & 24,9 & 719 & 30,9 & 1179 & 28,3 \\
\hline 65 y más & 1174 & 63,5 & 1313 & 56,5 & 2487 & 59,6 \\
\hline Total & 1848 & 100 & 2325 & 100 & $4173^{\star}$ & 100 \\
\hline
\end{tabular}


Tabla 6. Porcentajes y tasas estandarizadas de mortalidad de las principales localizaciones de muerte por cáncer en hombres y mujeres

Frequency and standardized death rates of the principal cancer sites, males and females

$\begin{array}{lcc} & \text { Porcentaje } & \text { tem }^{\star}(\mathrm{x} 100000) \\ \text { Hombres } & & \\ \text { Estómago } & 19,4 & 14,6 \\ \text { Próstata } & 16,5 & 10.9 \\ \text { Pulmón } & 7,8 & 5,9 \\ \text { Colon recto } & 6,4 & 4,7 \\ \text { Linfoma } & 5,9 & 4,5 \\ \text { Mujeres } & & \\ \text { Cuello uterino } & 17,1 & 14,0 \\ \text { Estómago } & 15,1 & 11,3 \\ \text { Mama } & 10,1 & 8,4 \\ \text { Colon recto } & 7,7 & 5,7 \\ \text { Pulmón } & 6,4 & 4,9\end{array}$

En Trujillo, Lima y Arequipa, la mortalidad por cáncer afectó más a mujeres. El Perú es de los pocos países junto con El Salvador y Nicaragua en los que la tasa de mortalidad global por cáncer es mayor en las mujeres, atribuible a las altas de incidencia de cáncer cervicouterino.

El cáncer cervicouterino ocupó el segundo lugar en la mortalidad total por cáncer en Trujillo. En
Cali, ${ }^{9}$ se ubicó en el cuarto lugar. En la mortalidad femenina por cáncer ocupó la primera localización en Trujillo, segunda en Arequipa y tercera en Lima. La mortalidad por cáncer cervical no ha declinado en Latino América como en los países industrialmente desarrollados probablemente por insuficiente cobertura del tamizaje en la población femenina de alto riesgo. Los programas de tamizaje en los países latinoamericanos generalmente están insertos en programas de planificación familiar y prenatal que sirven a poblaciones menores de 30 años con menor riesgo para desarrollar cáncer de cuello uterino ${ }^{(12)}$.

En Trujillo, el cáncer de próstata ocupó el tercer lugar de la mortalidad general por cáncer, en Arequipa el segundo lugar y el cuarto en Lima. En los hombres fue la segunda causa de muerte en Trujillo y Lima y la primera en Arequipa. En Sudamérica el cáncer de próstata ocupó la segunda localización de muerte por cáncer en Quito, tercera en Uruguay y Cali, cuarta en Argentina y el quinto en Valdivia. ${ }^{13}$ La mortalidad por cáncer colorrectal se ubicó en el cuarto puesto en Trujillo, la tasa de mortalidad es más alta que en Lima y Arequipa. En Argentina y Uruguay ocupó el segundo lugar, en Quito la cuarta localización.

El cáncer de bronquio y pulmón se ubicó en el quinto lugar de la mortalidad total por cáncer en Trujillo

Tabla 7. Frecuencia de la mortalidad por cáncer en la niñez

\begin{tabular}{lcccc} 
Childhood cancer mortality frequency & & & \\
Localización & niños & niñas & total & porcentaje \\
Leucemia & 16 & 11 & 27 & 42,8 \\
Sistema Nervioso Central & 8 & 7 & 15 & 23,8 \\
Linfoma & 3 & 2 & 5 & 8,0 \\
Hueso & 2 & 1 & 3 & 4,7 \\
Primario desconocido & 1 & 2 & 3 & 4,7 \\
Hígado & 0 & 2 & 2 & 3,2 \\
Tejidos blandos & 2 & 0 & 2 & 3,2 \\
Ovario & - & 2 & 2 & 3,2 \\
Ojo (retina) & 0 & 2 & 1 & 3,2 \\
Testículo & 1 & - & 1 & 1,6 \\
Riñón & 1 & 0 & 63 & 1,6 \\
\hline Total & 34 & 29 & 2 & 100
\end{tabular}


y Arequipa, el segundo lugar en Lima. En Argentina y Uruguay fue la primera causa de muerte. Entre los varones ocupó el tercer lugar en Trujillo, Lima y Arequipa y en mujeres el quinto lugar en Trujillo, cuarto lugar en Lima y el sexto en Arequipa.

Las tasas porcentuales de la mortalidad por cáncer en la niñez en Trujillo y Arequipa, fueron menores que en Lima. En Lima y Trujillo la leucemia fue la primera causa de muerte por cáncer en la niñez. La segunda causa fueron los tumores del sistema nervioso central. En Trujillo y Lima, la leucemia y linfoma juntos representaron el 50 por ciento de la mortalidad por cáncer en la niñez.

\section{CONCLUSIÓN}

Es preocupante que en Trujillo neoplasias malignas probadamente prevenibles, como el cáncer de cuello uterino y mama, juntos representen un tercio de la mortalidad por cáncer en la mujer. La política actual de control del cáncer está más orientada al tratamiento, que no ha modificado significativamente las altas tasas de enfermedad en etapas avanzadas ni la mortalidad. La estrategia de la prevención y diagnóstico temprano debiera ser prioritaria como política de salud para mejorar significativamente los indicadores de curación y disminución de la mortalidad por cáncer.

\section{REFERENCIAS BIBLIOGRÁFICAS}

I. Ferlay J, Shin HR, Bray F, Forman D, Mathers C, Parkin DM. Estimates of worldwide burden of cancer in 2008: Globocan 2008. Int J Cancer. 2010;127:2893-2917.

2. La mortalidad por cáncer está decayendo en algunos países de las Américas. OMS. Uruguay. 2014 El rincón de la Directora. [Página internet]. Accesado el 18/05/14 Disponible en: http:// www.paho.org/uru/index.php? option=comcontent\&view= article\&id $=775 \&$ ltemid $=$ I

3. Revilla L. Vigilancia epidemiológica de cáncer. Bol Epidemiol. (Lima). 20I I; 20(04):55-56.

4. Gerencia de Salud La Libertad. Defunciones registradas por grupos de causa básica de muerte:Trujillo. 2010.

5. Albújar PF. El cáncer en Trujillo, 1996-2002: incidencia y mortalidad. Registro de Cáncer de Base Poblacional de Trujillo, Perú. Informe N. ${ }^{\circ}$ 4. Trujillo; 2006

6. Cáncer en Lima Metropolitana 1994-1997. Registro de Cáncer de Lima Metropolitana.Vol. III. Lima. 2004.

7. Registro de Cáncer Poblacional de Arequipa 2002-2003. Arequipa. 2006.
8. Cueva P,Yépez J.eds. Epidemiología del cáncer en Quito 20032005. Registro Nacional de Tumores. Ecuador. 2006.

9. Instituto Nacional de Cancerología. Incidencia estimada y mortalidad por cáncer en Colombia. Colombia. [Página internet]. Accesado el 15/05//4 Disponible e $\mathrm{n}$ : www.cancer.gov.co.

10. Abriata G, Loria D. Análisis de mortalidad por cáncer en argentina 1980-2006. Boletín de Vigilancia de Enfermedades no Transmisibles y Factores de Riesgo N. ${ }^{\circ}$ I. Argentina. 2009. [Página internet]. Accesado el 17/05//4 Disponible en: http:// www.msal.gov.ar/inc/images/stories/downloads/publicaciones/ equipo_medico/Epidemiologia/Anlisis_de_la_mortalidad_por_ cncer_en_Argentina_1980-2006.pdf

II. Registro Nacional de Cáncer. Mortalidad por cáncer en Uruguay 201I. Uruguay. 2012. [Página internet]. Accesado el 18/05/14 Disponible en: www.comisioncancer.org.uy.

12. Robles SC,White F, Peruga A. Trends in cervical cancer mortality in the Americas. Bull Pan Am Health Organ. 1996;30(4):290-30I. 13. Registro Poblacional de Cáncer. Valdivia, Chile 2006-2008. Vol. N. ${ }^{\circ}$ 4. Chile. 20I I. [Página internet]. Accesado el 08/04/l4 Disponible en: www.siad-sps.cl

\section{Correspondencia}

Dr. Pedro Albújar Baca palbujarb@gmail.com

Fecha de recepción: 18 de mayo de 2014

Fecha de aceptación: 23 de junio de 2014

\section{Conflictos de interés}

El autor declara no tener conflictos de interés durante el planteamiento, ejecución de la investigación y la elaboración del artículo para su publicación. 